\title{
Prevalencia de Dirofilaria immitis en caninos domésticos de dos municipios del trópico de Guerrero, México.
}

Prevalence of Dirofilaria immitis in domestic canines from two municipalities in the tropic of Guerrero, Mexico.

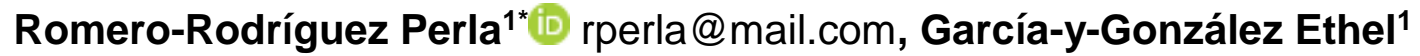 eth_cat@hotmail.com, Santos-Sotomaior Cristina2@ cristina.sotomaior@pucpr.br Pineda-Burgos Blanca1 ${ }^{1 D}$ uagro_blanca@hotmail.com, Olivar-Valladolid Gustavo1 mvzerickolivar@gmail.com, Hernández-Ruiz Pedro1@elhumogro@hotmail.com, Ponce-Covarrubias José ${ }^{{ }^{\star *}(\mathbb{D})}$ jlponce@uagro.mx}

${ }^{1}$ Escuela Superior de Medicina Veterinaria y Zootecnia No. 3, Universidad Autónoma de Guerrero, Tecpan de Galeana, Guerrero, México. ${ }^{2}$ Programa de Pós-Graduação em Ciência Animal, Escola de Ciências Agrárias e Medicina Veterinária - Pontifícia Universidade Católica do Paraná, São José dos Pinhais, Brasil. $\quad$ *Autor responsable: Perla Irasema Romero-Rodríguez. **Autor de correspondencia: José Luis Ponce-Covarrubias. Carr. Acapulco-Zihuatanejo km. 106+9000 Col. Las Tunas, C.P. 40900. Guerrero, México

\section{RESUMEN}

El objetivo del presente estudio fue determinar la prevalencia de Dirofilaria immitis (DI) en caninos domésticos en los municipios de Cuautepec y Acapulco de Juárez, Guerrero, México. Se usó muestreo por conglomerados de acuerdo a los domicilios seleccionados. Se obtuvieron muestras sanguíneas solo caninos mayores de un año de los dos municipios. En el estudio se encontró en el municipio de Cuautepec el $15.68 \%$ y en Acapulco de Juárez el $7.44 \%$ de caninos domésticos positivos a microfilarias. El mayor porcentaje de animales positivos se encontró en machos, en animales $>3$ años de edad y animales de talla chica y mediana en el municipio de Cuautepec $(P<0.05)$. Asimismo, en el municipio de Acapulco de Juárez se encontró un mayor porcentaje de hembras positivas, con edad $>3$ años de edad, de raza mestizo indefinido y que vivian en el exterior del hogar $(P<0.05)$. Se concluye que los caninos domésticos en los municipios de Cuautepec y Acapulco de Juárez, Guerrero se encontró una alta prevalencia de DI, y los machos tuvieron un mayor porcentaje en el municipio de Cuautepec y las hembras en Acapulco de Juárez.

Palabras clave: Nematodos gastrointestinales; infestación; corazón derecho; Dirofilaria immitis; sexo; muestras de sangre.

\begin{abstract}
The aim of the present study was to determine the prevalence of Dirofilaria immitis (ID) infection in domestic canines from Cuautepec and Acapulco de Juarez municipalities, in Guerrero Mexico. Cluster sampling was used, according to selected addresses. Blood samples were obtained from dogs over 1 year of age. In this study, $15.68 \%$ domestic canines in Cuautepec, and $7.44 \%$ in Acapulco de Juarez were positive to microfilariae. The highest percentage of positive animals was found in Cuautepec $(P<0.05)$ in males $>3$ years of age (small/medium-sized). Likewise, in Acapulco de Juarez, a higher positive percentage was found $(\mathrm{P}<0.05)$ in female $>3$ years old. It is concluded that in Cuautepec and Acapulco de Juarez Guerrero domestic canines have a high prevalence of DI, where males had the highest percentage in Cuautepec and females in Acapulco de Juarez.
\end{abstract}

Keywords: Gastrointestinal nematodes, infestation, right heart, Dirofilaria immitis, sex, bood samples. 


\section{INTRODUCCIÓN}

La filariosis canina es la enfermedad que da nombre a la infestación por el parásito Dirofilaria en perros, también conocida como dirofilariasis, verminosis cardiaca, enfermedad por gusanos cardiacos, enfermedad del gusano en el corazón (Rawlings y Calvert, 1997; Montoya-Alonso et al., 2011; Alho et al., 2018). En el mundo existen dos especies de Dirofilaria, de interés para la medicina humana y veterinaria $(D$. repens y D. immitis) (Liotta et al., 2013). La D. repens es una enfermedad presente en Europa, África y Asia; por su parte la DI o gusano del corazón presente en mamíferos, principalmente carnívoros y primates: perros, gatos, zorros, coyotes, hurones y leones marinos (Vezzani et al., 2006; Orihel et al., 1998; Genchi et al., 2011; Alho et al., 2018).

La DI es un nematodo cosmopolita, considerada originalmente de importancia veterinaria estricta; posteriormente fue reconocida de carácter zoonótico; en humanos causa lesiones cutáneas y pulmonares. Se han reportado casos de dirofilariosis en grandes vasos mesentéricos, peritoneales, cordón espermático y en corazón "derecho" (Chinapa et al., 2004; Sánchez-Klinge et al., 2011; Wang et al., 2019).

Los animales enfermos clínicamente presentan pocos signos de infestación; aunque estos dependen de la severidad de la enfermedad, la ubicación de la filaria, el tiempo que ha estado presente (Knight, 1980; Ceribasi y Simsek, 2012; Dearsley et al., 2019), y la cantidad de daños causados al corazón; así como a los pulmones, el hígado y otros órganos; pero siempre el animal afectado mostrará cada vez menos tolerancia al ejercicio (Fox et al., 1999; Ceribasi y Simsek, 2012; Wang et al., 2019). Los gusanos adultos, en el canino, forman una masa en el ventrículo derecho causando una falla cardiaca congestiva en la arteria pulmonar; mientras que las microfilarias circulan en la sangre (Knight, 1980; Fox et al., 1999; Montoya-Alonso et al., 2011; Alho et al., 2018).

La microfilaria circula en el torrente sanguíneo, pero no puede desarrollar gusanos adultos sin pasar por un huésped intermediario y transmisor, "el mosquito" hematófago que pertenecen al Phylum Arthropoda, Clase Insecta, Orden Diptera, Suborden Nematocera, Familia Culicidae (Christensen, 1978; Liotta et al., 2013; Alho et al., 2018) y los géneros: Aedes, Anopheles, Culex y Taeniohynchus; la familia tiene 3,000 especies incluidas en 34 géneros, receptivos como hospedadores intermediarios y vectores biológicos de DI (Sánchez-Klinge et al., 2011; Urquhart et al., 2001; Simón et al., 2012; Dearsley et al., 2019).

Para que ocurra el ciclo biológico en el mosquito, es necesario que en el mosquito infectado pase una serie de trasformaciones en estadios larvarios (L1, L2 y L3), todo esto sucede entre 13 a 16 días (Kittleson y Kienle, 2000; Bowman y Lynn, 1999; 
Montoya-Alonso et al., 2011; Dearsley et al., 2019). Por su parte, el desarrollo en el mamífero hospedador, ocurre después de 2 días hasta 3 meses, alcanzando longitudes de 3.2 a 11 cm (L4 y 5; Bowman y Lynn, 1999; Liotta et al., 2013; Wang et al., 2019). El número de gusanos adultos albergado varían de 1 a más de 250 en el perro (Dillon, 2000; Dearsley et al., 2019). La supervivencia de DI en el perro es de 3 a 8 años (Atkins, 1994; Dillon, 2000; Newton, 1968; Liotta et al., 2013; Dearsley et al., 2019).

En México se han realizado estudios epidemiológicos para determinar la presencia de DI en perros, y su prevalencia varía según las condiciones de vida de los animales y las variaciones climáticas de la región (Bautista-Garfias et al., 2001). En efecto, las mayores prevalencias se encuentran en lugares de regiones tropicales y subtropicales (Labarthe y Guerrero, 2005; Liotta et al., 2013; Wang et al., 2019). En un estudio de prevalencia realizado en los 11 municipios del estado de Nayarit, encontraron un rango de prevalencia de 2.5 a 33.33\% (González-Morteo et al., 2015; Wang et al., 2019). Rodríguez-Vivas et al. (1994), realizaron un estudio en la ciudad de Mérida, Yucatán, y encontraron una prevalencia de $12.5 \%$.

El examen sanguíneo es el método más práctico y sencillo para el diagnóstico de DI; aunque es una prueba cualitativa, no indica el número de microfilarias y la gravedad de las lesiones. En efecto, el diagnóstico se establece generalmente a partir de exámenes ordinarios de sangre (Paras, 2011; Wang et al., 2019), como la biometría hemática, casos de tos crónica en individuos que viven en áreas endémicas y para realizar detección de gusanos adultos en el corazón a través de placas radiográficas, especialmente en casos de síndrome de la vena cava (García et al., 2011; Alho et al., 2018). En animales enfermos se realiza un diagnóstico clínico por varios métodos para la identificación de microfilarias de perros infectados: a) método de observación directa del suero, b) método concentración sérico, c) métodos inmunológicos para detectar antígenos y d) técnica modificada de Knott (Ferrer-Montaño et al., 2002; Fernández et al., 2017; Dearsley et al., 2019).

En el estado de Guerrero y particularmente en los municipios de Acapulco de Juárez y Cuautepec no existe información sobre prevalencia de DI en caninos domésticos. Por lo anteriormente mencionado el objetivo del presente estudio fue determinar la prevalencia de Dirofilaria immitis en caninos domésticos en los municipios de Cuautepec y Acapulco de Juárez, Guerrero, México. 


\section{MATERIAL Y MÉTODOS}

\section{Área de estudio}

El presente estudio epidemiológico se realizó en los municipios de Cuautepec y Acapulco de Juárez, Guerrero, México. Esta región del estado pertenece al trópico y se

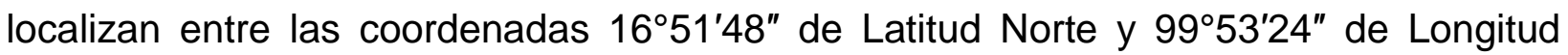
Oeste, con una altitud de entre 0 y 2,000 m.s.n.m. La temperatura y humedad ambiental promedio más alta $\left(39.5^{\circ} \mathrm{C}\right.$ y $\left.76 \%\right)$, se presentan durante los meses del verano y las más bajas $\left(16^{\circ} \mathrm{C}\right.$ y $\left.<70 \%\right)$ durante los meses del invierno (García, 1974).

\section{Animales}

Fueron muestreados al azar 196 caninos domésticos, 102 en el municipio de Cuautepec (41 machos y 61 hembras) y 94 Acapulco de Juárez (41 machos y 53 hembras). En ambos municipios el criterio de muestreo de animales fue de norte a sur y de este a oeste, tomando como referencia el centro de cada municipio. De los animales encontrados por visitas domiciliadas se muestrearon solo los mayores de un año, porque el ciclo biológico del parásito es largo y para el método de diagnóstico usado se requiere que tenga en su plasma microfilarias, alrededor de 7 meses después de la infestación (González-Morteo et al., 2015). Todos los procedimientos de muestreo y manejo de los animales reportados en el presente trabajo fueron de acuerdo a la Norma Oficial Mexicana con especificaciones técnicas de la producción, uso y cuidado de animales de laboratorio (SAGARPA, 2001).

\section{Recolección y preparación de las muestras}

Las muestras de sangre fueron tomadas en las horas comprendidas entre 18:00 20:00 h, por la periodicidad nocturna. Previa a la extracción de sangre se sujetó al animal para depilar la zona; con la respectiva antisepsia se extrajeron de la vena cefálica $3 \mathrm{ml}$ de sangre con anticoagulante (EDTA) y las muestras fueron conservadas de 4 a $6^{\circ} \mathrm{C}$ por 4 días para su posterior análisis.

Posteriormente, se realizaron frotis sanguíneos con la finalidad de identificar larvas de dirofilaria muertas. Para la técnica de Gota Gruesa se colocó una gota de sangre fresca sobre un portaobjetos, extendiéndola hasta cubrir una superficie doble de la original; finalmente, la preparación se dejó secar (al aire o en una estufa a $37^{\circ} \mathrm{C}$ ) y se observó al microscopio las huellas de la motilidad de las larvas. La técnica de Knott modificada se realizó mezclando $1 \mathrm{ml}$ de sangre con $9 \mathrm{ml}$ de formol al $2 \%$ en un tubo de vidrio; se centrifugó la mezcla durante 8 minutos a 1500 r.p.m.; se eliminó el sobrenadante, y se añadió al sedimento azul de metileno al $0.1 \%$, para luego examinar el sedimento al microscopio. 


\section{Variables de estudio}

El sexo, edad (1, 3 y $>3$ años), talla (chica, mediana, grande), raza (pura, mestizo indefinido), condición corporal (CC, escala: 1 a 5) (Laflamme, 1997), actividad o labor en el campo, arreo de ganado, cuidado del domicilio, ubicación (norte, sur, este u oeste) y posibles factores de riesgo como grado de urbanización de la coloniaviviendas donde habitaban los perros y exposición a mosquitos.

\section{Análisis estadísticos}

El sistema de muestreo por municipio Cuautepec y Acapulco de Juárez, fue por conglomerados de acuerdo a la ubicación de los domicilios muestreados (dinámica: norte a sur y de este a oeste). Se realizaron pruebas de contingencia en tablas $2 \times 2$; las proporciones entre variables fueron analizadas con la prueba de Chi-Cuadrada para establecer la correlación entre sexo y enfermedad. Todos los resultados fueron analizados con el programa estadístico SAS (2004).

\section{RESULTADOS Y DISCUSIÓN}

En el municipio de Cuautepec se encontró el $15.68 \%$ de caninos positivos a microfilarias. El mayor porcentaje de animales positivos se encontró en machos que en hembras y en animales $>3$ años de edad $(P<0.05)$. Los caninos que presentaron mayor porcentaje fueron los de CC de 4 y 5 que los menores a esta escala. Por su parte, se encontró un mayor porcentaje de animales positivos en los caninos de talla chica y mediana que de talla grande $(P<0.05)$. También se encontró un mayor porcentaje en caninos de raza mestizo indefinido, que vivían al exterior del hogar, en convivencia con otros congéneres y que dedicaban más tiempo al cuidado del hogar $(P<0.05)$ (Cuadro 1).

Los resultados del presente estudio muestran que en el municipio de Cuautepec se encontró una alta prevalencia de DI en caninos. Resultados consistentes con este estudio fueron similares a los encontrados en los municipios de Bahía de Banderas Nayarit (12.7\% prevalencia) (González-Morteo et al., 2015) y Chontalpa Tabasco (17.5\% prevalencia) (Torres-Chable et al., 2018); pero diferentes a los reportados por Rodríguez-Vivas et al. (1994) en el estado de Yucatán (6.54\% prevalencia). Estas mismas investigaciones (Rodríguez-Vivas et al., 1994; González-Morteo et al., 2015; Torres-Chable et al., 2018), mencionan no haber encontrado efecto entre los sexos (machos y hembras), edad, talla, raza, lugar donde habitaban (dentro o fuera de la vivienda) y convivencia con otros caninos.

Sin embargo, en el presente estudio los animales donde se encontró las microfilarias fueron machos, con alta CC (3 y 5 puntos), talla chica y mediana. Esto se puede explicar de la siguiente manera: los perros de trabajo generalmente son de raza mestizo indefinido, talla mediana, habitan en el exterior del hogar, tienen la posibilidad 
de interactuar más con otros caninos y debido a la ubicación están más expuestos a picaduras de mosquitos hospedadores del parásito DI.

\section{Cuadro 1. Frecuencia y porcentaje de caninos positivos a microfilaria de DI en el municipio de Cuautepec.}

\begin{tabular}{|c|c|c|c|c|}
\hline \multirow[t]{2}{*}{ Variable } & \multirow[t]{2}{*}{ Categoría } & \multirow{2}{*}{$\begin{array}{l}\text { Muestras } \\
(\mathrm{n})\end{array}$} & \multicolumn{2}{|c|}{ Positivos a microfilarias (Knott) } \\
\hline & & & Frecuencia & $\%$ \\
\hline \multirow[t]{2}{*}{ Sexo } & Hembra & 41 & $3^{a}$ & $7^{b}$ \\
\hline & Macho & 61 & $13^{b}$ & $21^{a}$ \\
\hline \multirow{3}{*}{ Edad (años) } & 1 & 36 & $4^{a}$ & $11^{\mathrm{b}}$ \\
\hline & 3 & 38 & $7^{a}$ & $18^{\mathrm{a}}$ \\
\hline & $>3$ & 28 & $5^{a}$ & $18^{\mathrm{a}}$ \\
\hline \multirow{3}{*}{${ }^{1} \mathrm{CC}$} & 1 a 2 & 24 & $1^{a}$ & $4^{c}$ \\
\hline & 3 & 49 & $8^{b}$ & $16^{b}$ \\
\hline & 4 a 5 & 29 & $7^{b}$ & $24^{a}$ \\
\hline \multirow{3}{*}{ Talla } & Chica & 22 & $3^{a}$ & $14^{\mathrm{b}}$ \\
\hline & Mediana & 77 & $13^{b}$ & $17^{\mathrm{b}}$ \\
\hline & Grande & 3 & $0^{a}$ & $0^{\mathrm{a}}$ \\
\hline \multirow[t]{2}{*}{ Raza } & Pura & 31 & $2^{a}$ & $6^{b}$ \\
\hline & Mestizo & 71 & $14^{b}$ & $20^{a}$ \\
\hline \multirow[t]{2}{*}{ Vivienda } & Interior & 16 & $0^{\mathrm{a}}$ & $0^{\mathrm{b}}$ \\
\hline & Exterior & 86 & $16^{b}$ & $19^{a}$ \\
\hline \multirow{2}{*}{ Convivencia } & Granja & 49 & $2^{a}$ & $4^{b}$ \\
\hline & Caninos & 53 & $14^{b}$ & $26^{a}$ \\
\hline \multirow[t]{2}{*}{ Actividad } & Hogar & 38 & $1^{\mathrm{a}}$ & $4^{b}$ \\
\hline & Campo & 64 & $5^{b}$ & $23^{a}$ \\
\hline \multirow{4}{*}{ Ubicación } & Norte & 26 & $3^{a}$ & $18^{c}$ \\
\hline & Sur & 22 & $7^{b}$ & $19^{c}$ \\
\hline & Este & 17 & $9^{b}$ & $24^{b}$ \\
\hline & Oeste & 37 & $7^{b}$ & $11^{a}$ \\
\hline \multirow[b]{2}{*}{ Microfilaria } & Negativa & 86 & $0^{a}$ & $0 \%^{a}$ \\
\hline & Positiva & 16 & $16^{b}$ & $15.65 \%$ b \\
\hline
\end{tabular}

Por otra parte, en el municipio de Acapulco de Juárez se encontró el $7.44 \%$ de los perros domésticos positivos a microfilarias. El mayor porcentaje de animales positivos se encontró en animales de talla chica, con CC de 1 a 3 y que convivían con congéneres en el hogar $(\mathrm{P}<0.05)$. Asimismo, se encontró mayor porcentaje de hembras positivas, con edad $>3$ años de edad, de raza mestizo indefinido y que vivían en el exterior del hogar $(\mathrm{P}<0.05$; Cuadro 2$)$.

Los resultados del presente estudio muestran que, en la ciudad de Acapulco de Juárez, Guerrero, se encontró una baja prevalencia de DI en perros domésticos. Resultados similares a los de este estudio fueron reportados por Rodríguez-Vivas et al. (1994) en el estado de Yucatán (6.54\% prevalencia). Contrario a estos resultados fueron 
diferentes a los encontrados en el municipio de San Blas Nayarit por González-Morteo et al. (2015; 33.33\% prevalencia) y por Torres-Chable et al. (2018) en Chontalpa Tabasco (17.5\% prevalencia). Estas mismas investigaciones (Rodríguez-Vivas et al., 1994; González-Morteo et al., 2015; Torres-Chable et al., 2018), mencionan no haber encontrado efecto entre los sexos (machos y hembras), edad, talla, raza, lugar donde habitaban (dentro o fuera de la vivienda) y convivencia con otros perros.

Sin embargo, en el presente estudio los animales donde se encontró las microfilarias fueron machos, de baja CC (1 a 3 puntos), talla chica y mediana; esto se puede explicar de la siguiente manera: la mayoría de mascotas son de talla mediana, habitan en el exterior del hogar, tienen la posibilidad de interactuar más con otros perros y tienen una mayor exposición a las picaduras de mosquitos hospedador intermediario del parásito.

En el presente estudio se puede mencionar que cuando menos en los municipios muestreados prevalece la enfermedad de DI; debido a esto y por algunas características particulares para considerarla como una enzootia a la enfermedad (Dirofilariasis). Como lo han considerado los autores mencionados en sus localidades; es posible emplear este término debido a que en esta zona geográfica especifica del estado de Guerrero las poblaciones caninas que viven en el exterior del hogar se ven afectadas continuamente por los mosquitos y consecuentemente por DI.

\section{Cuadro 2. Frecuencia y porcentaje de caninos positivos a microfilaria de DI en la ciudad de} Acapulco.

\begin{tabular}{|c|c|c|c|c|}
\hline \multirow[t]{2}{*}{ Variable } & \multirow[t]{2}{*}{ Categoría } & \multirow[t]{2}{*}{$\mathrm{n}$} & \multicolumn{2}{|c|}{ Positivos a microfilarias (Knott) } \\
\hline & & & Frecuencia & $\%$ \\
\hline \multirow{3}{*}{ Sexo } & Hembra & 53 & $6^{a}$ & $11 \%^{a}$ \\
\hline & Macho & 41 & $1^{b}$ & $2 \% b$ \\
\hline & 8 a 12 & 40 & $3^{a}$ & $8 \%{ }^{a}$ \\
\hline \multirow[t]{3}{*}{ Edad (meses) } & 13 a 36 & 36 & $2^{a}$ & $6 \% a$ \\
\hline & $>37$ & 19 & $2^{a}$ & $11 \% b$ \\
\hline & 1 a 3 & 50 & $6^{a}$ & $12 \%{ }^{a}$ \\
\hline \multirow{2}{*}{ Escala de CC } & 3 a 5 & 44 & $1^{b}$ & $2 \% \mathrm{~b}$ \\
\hline & Chica & 36 & $6^{a}$ & $17 \% a$ \\
\hline \multirow[t]{3}{*}{ Talla } & Mediana & 42 & $0^{\mathrm{b}}$ & $0 \% \mathrm{~b}$ \\
\hline & Grande & 16 & $1^{\mathrm{b}}$ & $6 \% \mathrm{~b}$ \\
\hline & Definida & 21 & $0^{\mathrm{a}}$ & $0 \% \mathrm{a}$ \\
\hline \multirow{2}{*}{ Raza } & Mestiza indefinida & 73 & $7^{\mathrm{b}}$ & $10 \% \mathrm{~b}$ \\
\hline & Interior & 12 & $0^{\mathrm{a}}$ & $0 \% \mathrm{a}$ \\
\hline \multirow[t]{2}{*}{ Hábitat } & Exterior & 82 & $7^{\mathrm{b}}$ & $9 \%$ b \\
\hline & No & 34 & $0^{\mathrm{a}}$ & $0 \%{ }^{\mathrm{a}}$ \\
\hline \multirow[t]{2}{*}{$\begin{array}{c}\text { Convivencia con } \\
\text { animales }\end{array}$} & Perros & 60 & $7^{b}$ & $12 \%{ }^{b}$ \\
\hline & Negativa & 87 & $0^{a}$ & $0 \%{ }^{a}$ \\
\hline Microfilaria & Positiva & 7 & $7^{b}$ & $7.44 \%$ b \\
\hline
\end{tabular}




\section{CONCLUSIÓN}

Los resultados del presente estudio permiten concluir que, en los caninos domésticos de los municipios de Cuautepec y Acapulco de Juárez, Guerrero, se encontró una alta prevalencia de DI (promedio: 11.56\%), y los machos tuvieron un mayor porcentaje de infestación (21\%; Cuautepec). Asimismo, se encontró que los caninos de talla mediana y que presentaron CC alta dieron positivos en las pruebas de detección del parásito.

\section{AGRADECIMIENTOS}

Los datos que se presentan en el presente manuscrito fueron parte de la tesis de José Carlos Cisneros. Se agradece al Dr. Abner Gutiérrez, del departamento de Agronomía División de Ciencias de la Vida, Campus Irapuato - Salamanca, Universidad de Guanajuato, por todas las facilidades en el análisis de las muestras. Se agradece a todos los miembros del grupo de investigación "Sistemas de Producción Animal" de la Escuela Superior de Medicina Veterinaria y Zootecnia No. 3, Universidad Autónoma de Guerrero.

\section{LITERATURA CITADA}

ALHO AM, Mireles J, Schnyder M, Cardoso L, Belo S, Deplazes P, Carvalho. 2018. Dirofilaria immitis and Angiostrongylus vasorum: The current situation of two major canine heartworms in Portugal. Veterinary Parasitology. 252:120-126. doi: 10.1016/j.vetpar.2018.01.008.

ATKINS CE. 1994. "Síndrome de Dirofilariosis de la Cava". En: Kirk RW, Bonagura J, Terapéutica Veterinaria de Pequeños Animales. Madrid, España: Editorial Interamericana McGraw-Hill. 802 p. ISBN: 9788448603533.

BAUTISTA-Garfias CR, Arroyo-Rojas M, Velasco-Castrejón O, Canto-Ortiz L. 2001. Comparación de las pruebas quantitative buffy coat, frotis grueso de sangre y observación directa para el diagnóstico de la infección por Dirofilaria immitis en perros de tres zonas geográficas de México. Veterinaria México. 32(2):153-156. ISSN: 24486760 .

BOWMAN DD, Lynn RC. 1999. Georgis' Parasitology for Veterinarians. 7th ed., W.B. Saunders, Philadelphia. USA. https://www.elsevier.com/books/georgis-parasitology-forveterinarians/bowman/978-1-4557-4006-2

CERIBASI AO, Simsek S. 2012. Histopathologic Effects of Dirofilaria immitis Microfilaria on Intestinal organs of dog confirming by PCR Technique. Iran Journal Parasitology. 7(2):103-107.

CHIPANA C, Chávez AV, Casas E, Suárez F. 2014. Frecuencia de dirofilaria immitis en caninos del distrito de San Juan de Lurigancho. Revista de Investigaciones Veterinarias del Perú. 15(2):141-144. http://www.scielo.org.pe/pdf/rivep/v15n2/a08v15n2 
CHRISTENSEN BM. 1978. Dirofilaria immitis: effect on the longevity of Aedes trivittatus. Experimental Parasitology. 44:116-123. doi.org/10.1016/0014-4894(78)90087-5

DEARSLEY EJ, O'Handley RM, Caraguel CGB. 2019. Is canine heartworm (Dirofilaria immitis) endemic to South Australia?. Australia's Premier Veterinary Science Text. 97(6):191-196. doi: 10.1111/avj.12814.

DILLON R. 2000. Dirofilariosis in dogs and cats. En: ETTINGER, S. J., E. C. Feldman. 2000. Textbook of veterinary internal medicine. Disease of the dog and cat. 5th ed., W. B. Saunders Company, Philadelphia. USA. https://www.worldcat.org/title/textbook-ofveterinary-internal-medicine-diseases-of-the-dog-and-the-cat/oclc/428770833

FERNÁNDEZ K, Ayora P, Muñoz T. 2017. Diagnóstico de Dirofilaria inmitis en perros de la ciudad de Guayaquil mediante tres métodos de laboratorio. Centro de Biotecnología. 6:41-47.

FERRER-Montaño JA, Árraga de Alvarado CM, Alvarado-Morillo M, Sandoval-Martínez JE. 2002. Diagnóstico de Dirofilariosis: un estudio comparativo usando las pruebas de ELISA y de WOO. Revista Científica, FCV-LUZ. 5:351-357.

FOX R, Sisson D, Moïse N. 1999. Textbook of Canine and Feline Cardilogy. Principles and Clinical Practice. 2 edition. Saunders, Philadelphia. USA.

GARCÍA E. 1973. Modificaciones al sistema de clasificación climática de Köeppen. 2da edn. Instituto de Geografía, Universidad Nacional Autónoma de México, México, D.F, México. 11-90p.

GARCíA Herrera RA, Torres Chable DM, Peralta Torres JA, Thomas TJA. 2011. Determinación de la prevalencia de micro filariosis en perros de Tabasco, México. XX Congreso Nacional Sociedad Mexicana Patología Veterinaria. 274p.

GENCHI C, Mortarini M, Rinaldi L, Cringoli G, Traldi G, Genchi M. 2011. Hanging climate and changing vector-borne disease distribution: The example of Dirofilaria in Europe. Veterinary Parasitology. 176(4):295-299. doi: 10.1016/j.vetpar.2011.01.012

GONZÁLEZ-Morteo C, De la Cruz-Moreno O, Álvarez-Guerrero C, Peña-Parra B, Carrillo-Díaz F, Borrayo-González J. 2015. Prevalencia de Dirofilaria immitis en 11 municipios de Nayarit. Abanico Veterinario. 5(2):42-48.

KITTLESON MD, Kienle RD. 2000. Medicina cardiovascular de pequeños animales. 2a ed., Multimédica, Barcelona. España. https://latam.casadellibro.com/libro-medicinacardiovascular-de-pequenos-animales/9788492342792/749385

KNIGHT DH. 1980. Evolution of pulmonary artery disease in canine dirofilariasis: Evaluation by blood pressure measurements and angiography. Proceeding of the 
Heartworm Symposium 80. Bonner Springs, Kansas. 1980:55-62. Veterinary Medicine Publishing. Bonner Springs, Kansas (1980).

LABARTHE N, Guerrero J. 2005. Epidemiology of heartworm: What is happening in South America and Mexico? Veterinary Parasitology. 133:149-156. doi: 10.1016/j.vetpar.2005.04.006

LAFLAMME D. 1997. Development and validation of a body condition score system for dogs. Canine Practice. 22(4):10-15. http://agris.fao.org/agrissearch/search.do?recordID=US9742264

LIOTTA JL, Sandhu GK, Rishniw M, Bowman DD. 2013. "Differentiation of the Microfilariae of Dirofilaria immitis and Dirofilaria repens in Stained Blood Films". Journal of Parasitology. 99(3):421-425. doi: 10.1645/12-10.1

MONTOYA-Alonso JA, Carretón E, Corbera JA, Juste MC, Mellado I, Morchón R, Simón F. 2011. Current prevalence of Dirofilaria immitis in dogs, cats and humans from the island of Gran Canaria, Spain. Veterinary Parasitology. 176(4):291-294. doi: 10.1016/j.vetpar.2011.01.011

NEWTON WL. 1968. Longevity of an experimental infection with Dirofilaria immitis in a dog. Journal Parasitology. 54:187-188. doi: 10.2307/3276912

ORIHEL T, Eberhard M. 1998. Zoonotic filariasis. Clinical Microbiology Reviews. 11: 366-381. https://www.ncbi.nlm.nih.gov/pmc/articles/PMC106837/pdf/cm000366.pdf

PARAS KL. 2011. Epizootiology of dog Heartworm Dirofilaria immitis in Oklahoma (Tesis de Maestría). Oklahoma, USA: Oklahoma State University Stillwater. 2011:76.

RAWLINGS CA, Calvert CA. 1997. Verminosis cardiaca. En: ETTINGER SJ, FELDMAN EC. 1997. Tratado de Medicina Interna Veterinaria. 4a ed., Inter-Médica, Buenos Aires. Argentina.

RODRíGUEZ-Vivas RI, Domínguez AJL, Solís RFA, Cob GLA. 1994. Prevalencia de Dirofilaria immitis en perros callejeros de la ciudad de Mérida, Yucatán, México. Veterinaria México. 25:145-148.

SAGARPA 2001. Norma Oficial Mexicana NOM-062-ZOO-1999, Especificaciones técnicas para la producción, cuidado y uso de los animales de laboratorio. Secretaría de Agricultura, Ganadería, Desarrollo Rural, Pesca y Alimentación. Diario Oficial de la Federación, 22 de Agosto de 2001. http://www.sagarpa.gob.mx/normateca/Normateca/SENASICA\%20NORM .\%20143.pdf. Consultado 18 enero de 2019. 
SÁNCHEZ-Klinge LE, Calvo RP, Mutis BCA. 2011. Dirofilaria immitis: una zoonosis presente en el mundo. Revista Medicina Veterinaria. 22:57-68. http://www.scielo.org.co/pdf/rmv/n22/n22a07.pdf

SAS (ed) (2004) SAS/STAT: User's guide statistics released 9.1, 2nd edn. SAS Institute, Inc., Cary

SIMÓN F, Siles-Lucas M, Morchón R, González-Miguel J, Mellado I, Carretón E, Montoya-Alonso JA. 2012. Human and animal dirofilariasis: the emergence of a zoonotic mosaic. Clinical Microbiology Reviews. 25:507-544. doi: 10.1128/CMR.0001212.

TORRES-Chable OM, Baak-Baak CM, Cigarroa-Toledo N, Blitvich NJ, Brito-Argaez LG, Alvarado-Kantun YN, Zaragoza-Vera CV, Arjona-Jimenez G, Moreno-Perez LG, Medina-Perez P, Machain-Williams Cl, Garcia-Rejon JE. 2018. Molecular detection of Dirofilaria immitis in dogs and mosquitoes in Tabasco, Mexico. J Vector Borne Dis. 2018;55:151-158. doi:10.4103/0972-9062.242563.

URQUHART GM, Armour J, Duncan JL, Dunn AM, Jenningns FW. 2001. Parasitología Veterinaria. 2 ed. Zaragoza, ES. Acribia. 355p.

VEZZANI D, Carbajo AE. 2006. Spatial and temporal transmission risk of Dirofilaria immitis in Argentina. International Journal for Parasitology. 26:1463-1472. doi:10.1016/j.jpara.2006.08.012.

WANG J, Zhu X, Ying Z, Han Q, Liao C, Wang J, Zhao J, Sun J, Linsay DS. 2019. Prevalence of Ditofilaria immitis infections in dogs and cats in Haunan Island/Province and Three other coastal cities of China base don antigen testing and PCR. Journal of Parasitology. 105(2):199-202. doi: 10.1645/18-164. 